\title{
Unchanged expression of the sodium-dependent phosphate cotransporter NaPi-IIa despite diurnal changes in renal phosphate excretion
}

\author{
Bielesz, B ; Bacic, D ; Honegger, Katharina ; Biber, J ; Murer, H ; Wagner, C A
}

\begin{abstract}
Renal phosphate excretion is subjected to circadian rhythmicity. The bulk of filtered inorganic phosphate $(\mathrm{P}(\mathrm{i}))$ is reabsorbed by the sodium-dependent phosphate cotransporter NaPi-IIa. The regulation of proximal tubular phosphate reabsorptive capacity is largely attributed to the altered abundance of $\mathrm{NaPi}$-IIa residing in the brush border membrane (BBM) of proximal tubular cells. Therefore, we examined if the diurnal rise in renal phosphate excretion is accompanied by a corresponding change in NaPi-IIa expression. Renal phosphate excretion, creatinine clearance, and serum phosphate were determined at consecutive time points in rats, starting from 8 a.m. until 5 p.m. During this period, renal phosphate excretion (fractional $\mathrm{P}(\mathrm{i})$ excretion) increased more than eightfold until 5 p.m. compared to the morning values at 8 a.m. In addition, serum phosphate and creatinine clearance as well as the calculated tubular phosphate threshold increased. Neither immunoblot analysis of BBMs nor immunohistochemical staining for NaPi-IIa yielded evidence for a lower abundance of NaPi-IIa in kidneys collected in the afternoon compared to those in the morning. However, kidneys sampled in the afternoon showed a small decrease $(14 \%)$ in (32)P uptakes into BBM vesicles (BBMVs). Thus, the diurnal rise in renal phosphate excretion was associated with a mild reduction in the sodium-dependent phosphate transport rate in proximal tubular BBMs. There was no apparent downregulation of NaPi-IIa abundance and only a small reduction in $\mathrm{Na}(+)$-dependent Pi-transport activity. Thus, the diurnal changes in urinary phosphate excretion appear to be mainly related to changes in serum phosphate and tubular threshold but not to NaPi-IIa expression.
\end{abstract}

DOI: https://doi.org/10.1007/s00424-006-0087-0

Posted at the Zurich Open Repository and Archive, University of Zurich

ZORA URL: https://doi.org/10.5167/uzh-1346

Journal Article

Published Version

Originally published at:

Bielesz, B; Bacic, D; Honegger, Katharina; Biber, J; Murer, H; Wagner, C A (2006). Unchanged expression of the sodium-dependent phosphate cotransporter NaPi-IIa despite diurnal changes in renal phosphate excretion. Pflügers Archiv: European Journal of Physiology (Pflugers Archiv), 452(6):683689.

DOI: https://doi.org/10.1007/s00424-006-0087-0 


\section{Bernhard Bielesz - Desa Bacic - Katharina Honegger • Jürg Biber · Heini Murer · Carsten A. Wagner \\ Unchanged expression of the sodium-dependent phosphate cotransporter NaPi-lla despite diurnal changes in renal phosphate excretion}

Received: 31 August 2005 / Accepted: 10 April 2006 / Published online: 19 May 2006

C) Springer-Verlag 2006

\begin{abstract}
Renal phosphate excretion is subjected to circadian rhythmicity. The bulk of filtered inorganic phosphate $\left(\mathrm{P}_{\mathrm{i}}\right)$ is reabsorbed by the sodium-dependent phosphate cotransporter NaPi-IIa. The regulation of proximal tubular phosphate reabsorptive capacity is largely attributed to the altered abundance of NaPi-IIa residing in the brush border membrane (BBM) of proximal tubular cells. Therefore, we examined if the diurnal rise in renal phosphate excretion is accompanied by a corresponding change in NaPi-IIa expression. Renal phosphate excretion, creatinine clearance, and serum phosphate were determined at consecutive time points in rats, starting from 8 a.m. until 5 p.m. During this period, renal phosphate excretion (fractional $\mathrm{P}_{\mathrm{i}}$ excretion) increased more than eightfold until 5 p.m. compared to the morning values at 8 a.m. In addition, serum phosphate and creatinine clearance as well as the calculated tubular phosphate threshold increased. Neither immunoblot analysis of BBMs nor immunohistochemical staining for NaPi-IIa yielded evidence for a lower abundance of $\mathrm{NaPi}$-IIa in kidneys collected in the afternoon compared to those in the morning. However, kidneys sampled in the
\end{abstract}

B. Bielesz $\cdot$ K. Honegger $\cdot$ J. Biber $\cdot$ H. Murer $\cdot$ C. A. Wagner Institute of Physiology and Center for Integrative Human Physiology (CIHP), University of Zurich,

Winterthurerstrasse 190,

8057 Zurich, Switzerland

e-mail: Wagnerca@access.unizh.ch

Tel.: +41-44-6350659

Fax: +41-44-6350814

D. Bacic

Institute of Anatomy, University of Zurich,

Winterthurerstrasse 190,

8057 Zurich, Switzerland

B. Bielesz $(\bowtie)$

Department of Internal Medicine III, Division of Nephrology, Medical University of Vienna,

Währinger Gürtel 18-20,

1090 Vienna, Austria

e-mail: bernhard.bielesz@meduniwien.ac.at

Tel.: +43-1-404005526

Fax: $+43-1-404004452$ afternoon showed a small decrease $(14 \%)$ in ${ }^{32} \mathrm{P}$ uptakes into BBM vesicles (BBMVs). Thus, the diurnal rise in renal phosphate excretion was associated with a mild reduction in the sodium-dependent phosphate transport rate in proximal tubular BBMs. There was no apparent downregulation of NaPi-IIa abundance and only a small reduction in $\mathrm{Na}^{+}$-dependent Pi-transport activity. Thus, the diurnal changes in urinary phosphate excretion appear to be mainly related to changes in serum phosphate and tubular threshold but not to NaPi-IIa expression.

Keywords Sodium-dependent phosphate cotransporter . Diurnal changes $\cdot$ Renal phosphate excretion

\section{Introduction}

Circadian variations in urinary phosphate excretion and serum phosphate are long known in humans $[1,4,9,11,12$, $16,29]$ and reviewed in the study of Mills [17]. Serum phosphate is reported to be lowest in the morning around 7-10 a.m. then rises to reach a first peak at late afternoon and a second at early morning hours around $2-3$ a.m. Renal phosphate excretion roughly parallels this pattern $[12,17]$. Circadian rhythmicity of both phosphate excretion and serum phosphate is thought to consist of an endogenous component that is strongly modulated by exogenous influences such as night shifts, sleep, exercise, or food intake (reviewed in the work of Mills [17]). Such diurnal variation has also been reported in rats but the reported serum and urinary excretion pattern for phosphate varied under different experimental conditions [14, 24, 26, 27]. Shinoda and Seto [26] reported a low morning (9 a.m.) level of serum phosphate with a forenoon rise and a peak at 5 p.m. in Wistar rats on a 12:12 h light-dark cycle and food and water ad libitum. The afternoon peak was followed by a fall to morning levels during the night hours. The authors also demonstrated that, consistent with the findings in humans, daily serum phosphate curves were altered considerably under continuous light exposure or food restriction. The precise mechanisms, however, by which 
the kidney exerts diurnal changes in renal phosphate excretion, are not known.

It is well established that the sodium-dependent phosphate cotransporter NaPi-IIa (SLC34A2) is the major quantitative key player in renal phosphate reabsorption and its regulation (reviewed in the works of Murer and coworkers [19, 21-23]). The majority of physiological and pathophysiological alterations in renal phosphate excretion can be ascribed to closely corresponding changes in NaPi-IIa protein abundance in the brush border membrane (BBM) of proximal tubular cells. In kinetic terms, this is reflected by changes in $V_{\max }$ of sodium-dependent phosphate transport. The amounts of dietary phosphate intake and parathyroid hormone (PTH) are two well-studied examples for such physiological regulators (reviewed in the work of Murer et al. [21]). Given the crucial importance of altered BBM expression in renal phosphate maintenance, we examined if diurnal changes in renal phosphate excretion can be attributed to corresponding variations in NaPi-IIa protein abundance.

\section{Materials and methods}

Animals

Male Wistar rats weighing 200-280 g were used for the experiments. Animals were allowed to adapt after purchase for a minimum of 1 week and were kept on a $12 \mathrm{~h}$ lightdark cycle (lights on from 7 a.m. to 7 p.m.). Before and throughout the experiments, rats had free access to normal rodent chow containing 1,000 IU/kg Vitamin D and 0.8\% phosphorus (Provimi Kliba SA, Kaiseraugst, Switzerland) and water ad libitum. All studies were according to Swiss Animal Welfare Laws and approved by the local authority (Veterinäramt Zürich).

\section{Experimental procedures}

To estimate changes in glomerular filtration rate during our experiments, we measured total volumes of each urine sample in eight rats. The experiments were carried out in metabolic cages; food and water were provided ad libitum. The experiment was started at 7 a.m. to empty the bladder from overnight urine, and the first morning urine samples were collected at approximately 9 a.m. in all rats. Additional samples were taken every 90-150 min until late afternoon $(5$ p.m.). About $5-\mu l$ concentrated hydrochloric acid per milliliter of urine was added to the samples before storing them at $-20^{\circ} \mathrm{C}$ for later analysis.

Urine volume was measured by weighing; residual urine was collected with a dry tissue which was also weighed. Collection time started after taking the first sample, volumes of all subsequent samples were thoroughly measured, and collection times were documented. Blood samples were collected from the tail vein using heparinized syringes at approximately 9 a.m., 1 p.m., and 5 p.m., and serum was rapidly separated by centrifugation. For determination of creatinine clearance and filtered phosphate load at 11 a.m. and 3 p.m., the means of serum creatinine and phosphate at 9 a.m./1 p.m. and 1 p.m. $/ 5$ p.m., respectively, were used for calculation.

Urine and serum analysis

Serum and urinary concentrations of phosphate $\left(\mathrm{P}_{\mathrm{i}}\right)$ and creatinine (Crea) were determined using commercial kits (Sigma Diagnostics, Munich, Germany, and Wako Chemicals, Reinach, Switzerland) according to the manufacturers' protocols. Renal fractional phosphate excretion was calculated using the formula ( $u$...urine, $s$...serum):

$F E_{P i}(\%)=\frac{\text { phosphate }_{U} \times \text { creatinine }_{S}}{\text { creatinine }_{U} \times \text { phosphate }_{S}} \times 100$.

Creatinine clearance $(\mathrm{CCl})$, filtered phosphate load $\left(\mathrm{FP}_{i}\right)$, and the endogenous (without phosphate loading) tubular threshold for phosphate $(\mathrm{Tp} / \mathrm{CCl})$ were calculated by using the following formulas [3]:

$$
\begin{aligned}
& C C l=\frac{\text { volume }_{U} \times \text { creatinine }_{U}}{\text { creatinine }_{S} \times \text { time }} \\
& F P_{i}=C C l \times \text { phosphate }_{S} \\
& T p / C C l=\text { phosphate }_{S}-\left(\text { phosphate }_{U} \times \frac{\text { creatinine }_{S}}{\text { creatinine }_{U}}\right)
\end{aligned}
$$

Preparation of BBMVs and ${ }^{32} \mathrm{P}$ uptakes

In separate experiments, four rats per day were killed under isoflurane anesthesia, two at 8 a.m. and two at 3 p.m. Kidneys were rapidly removed, shock-frozen in liquid nitrogen, and stored at $-80^{\circ} \mathrm{C}$ until further analysis. Urine and serum samples were also taken to confirm the expected changes in renal phosphate excretion. BBMVs were prepared from whole rat kidney using the $\mathrm{Mg}^{2+}$ precipitation technique as described previously [2]. The phosphate transport rate into BBMVs was measured in freshly prepared $\mathrm{BBMV}$ at $25^{\circ} \mathrm{C}$ in the presence of inward gradients of $100 \mathrm{mM} \mathrm{NaCl}$ or $100 \mathrm{mM} \mathrm{KCl}$ and $0.1 \mathrm{mM}$ K-phosphate (kidney BBMVs) as described [20]. Phosphate uptake was determined after $60 \mathrm{~s}$, representing initial linear conditions, and after $120 \mathrm{~min}$, to determine the equilibrium values. Total protein concentration was measured using the Bio-Rad Protein Assay kit, Bio-Rad, Hercules, CA, USA. BBMVs were stored at $-80^{\circ} \mathrm{C}$ until further use. 
Immunoblotting and quantification of NaPi-IIa protein in renal BBMVs

Twenty micrograms of BBM protein was solubilized in Laemmli sample buffer [15], and sodium dodecyl sulfate polyacrylamide gel electrophoresis (SDS-PAGE) was performed using $10 \%$ polyacrylamide gels. For immunoblotting, proteins were transferred electrophoretically from gels to PVDF-membranes (Immobilon-P, Millipore, Bedford, MA, USA) [28]. After blocking with $5 \%$ milk powder in Trisbuffered saline $/ 0.5 \%$ Triton X-100 for 60 min, the blots were incubated with the primary antibodies (rabbit anti-NaPi-IIa serum 1:6,000 [5] and mouse monoclonal anti-actin (42 kDa, Sigma) 1:5,000), either for $2 \mathrm{~h}$ at room temperature or overnight at $4{ }^{\circ} \mathrm{C}$. After washing and subsequent blocking, blots were incubated for $1 \mathrm{~h}$ at room temperature with the secondary IgG antibodies (donkey anti-rabbit 1:12,000 or sheep anti-mouse 1:12,000, respectively), conjugated with horseradish peroxidase (GE-Amersham Life Sciences, Piscataway, NJ, USA). Antibody binding was detected with the peroxidase/luminal enhancer kit (Pierce, Rockford, IL, USA) with a camera and analyzed using AIDA Imaging software (Diana III; Raytest, Straubenhardt, Germany) to calculate the $\mathrm{NaPi}-\mathrm{II} /$ actin ratio.

Immunohistochemistry

Anesthetized rats were fixed by vascular perfusion via the abdominal aorta, at a pressure of 0.4 bar, as described previously [6]. The fixative consisted of $3 \%$ paraformaldehyde, $0.1 \%$ glutaraldehyde, and $0.05 \%$ picric acid in $0.1 \mathrm{M}$ cacodylate buffer ( $\mathrm{pH} 7.4$; containing $3 \mathrm{mM} \mathrm{MgCl} 2$ and adjusted to $300 \mathrm{mosM} / \mathrm{l}$ with sucrose) and $4 \%$ hydroxyethyl starch in saline (HAES steril; Fresenius, Stans, Switzerland). After $5 \mathrm{~min}$, the fixative was washed out by perfusion with a hydrostatic pressure of $70 \mathrm{~cm}$ for 5 min with cacodylate buffer. Perfusion-fixed kidneys were cut into slices, mounted onto thin cork plates, and immediately frozen in liquid propane cooled with liquid nitrogen. For NaPi-IIa immunofluorescence staining, $4-\mu \mathrm{m}$-thick cryosections mounted on chromalum/gelatincoated glass slides were pretreated for 10 min with $3 \%$ defatted milk powder and $0.02 \%$ Triton X-100 ("blocking solution") in phosphate-buffered saline (PBS). After rinsing with PBS, sections were incubated with rabbit anti-rat polyclonal antiserum against the NaPi-IIa protein diluted 1:500 in "blocking solution" (PBS with 0.1\% BSA containing $0.02 \%$ Triton X-100). After overnight incubation at $+4^{\circ} \mathrm{C}$, sections were rinsed three times with PBS and covered for $45 \mathrm{~min}$ at room temperature in the dark with swine anti-rabbit IgG conjugated to fluorescein isothiocyanate (Dakopatts, Glostrup, Denmark) diluted 1:50 in PBS/ milk powder. Double stainings of NaPi-IIa and $\beta$-actin filaments were achieved by adding rhodamine-phalloidin (Molecular Probes, Eugene, OR, USA) at a dilution 1:50 in the solution containing secondary antibodies. Finally, the sections were rinsed three times with PBS, covered with a glass slip using DAKO-Glycergel (Dakopatts) containing $2.5 \%$ 1,4-diazabicyclo [2.2.2.] octane (Sigma, St. Louis,
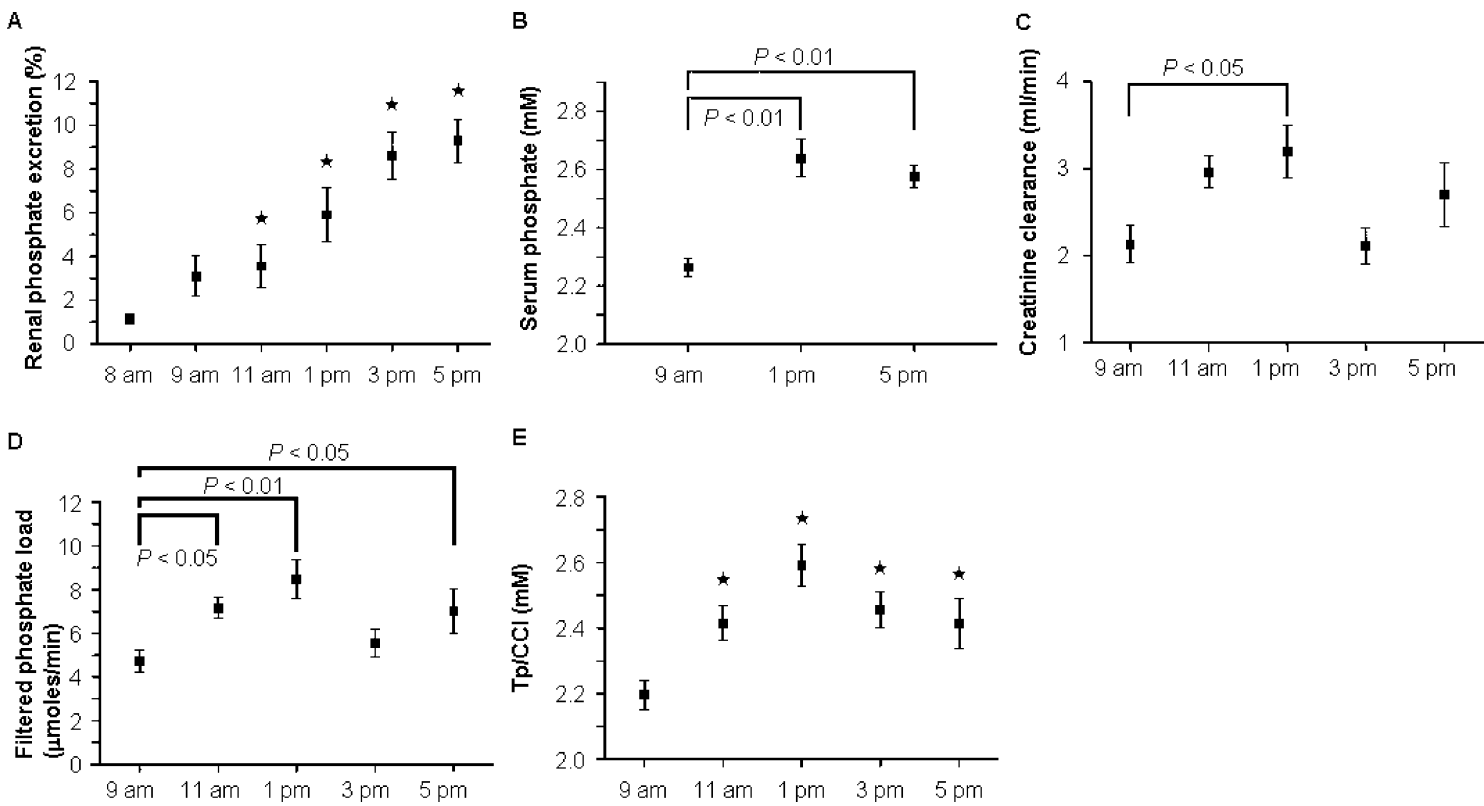

E

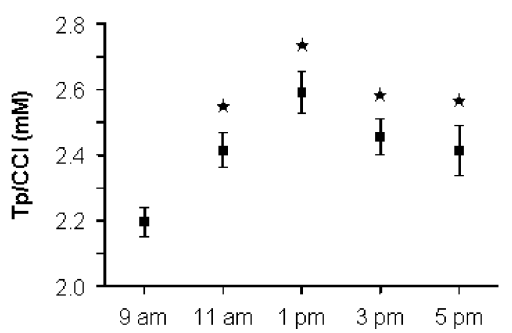

Fig. 1 Time course of renal phosphate handling. a Renal phosphate excretion $\left[\mathrm{FE}_{\mathrm{Pi}}(\%)\right]$ in male Wistar rats was lowest at 8 a.m. and increased steadily until 5 p.m. Asterisks indicate significant differences vs the first morning point; 8 a.m. vs 11 a.m.: $p<0.05$; 8 a.m. vs 1 p.m., 3 p.m., 5 p.m. $p<0.01 ; n=16$. b Serum phosphate concentrations rose from 9 a.m. to 1 p.m. and remained stable until 5 p.m. c Creatinine clearance increased from the first measurement
(9 a.m.) until 1 p.m. and decreased thereafter again, $n=8$. d The filtered phosphate load was calculated from the serum phosphate concentration and creatinine clearance and increased from morning to afternoon. e The tubular threshold for phosphate could also be calculated and showed a similar time course as the filtered phosphate load. Asterisks indicate a significant change compared to 9 a.m.; $p<0.01 ; n=8$ 
MO, USA) as a fading retardant, and studied with an epifluorescence microscope.

\section{Statistics}

Numerical data are presented as mean \pm standard error unless otherwise stated. All data were tested for statistically significant differences using the paired and unpaired student's $t$ test, ANOVA, and Dunnett's multiple comparison test. Only data with $p \leq 0.05$ were considered as statistically significantly different.

\section{Results}

Diurnal increase in renal phosphate excretion

Under the conditions described in the "Materials and methods" section, we observed in the morning (8 a.m.) a fractional renal phosphate excretion of $1.1 \pm 0.2 \%$. After a gradual increase throughout the day, the fractional renal phosphate excretion reached $9.3 \pm 1 \%$ in the late afternoon at 5 p.m. (Fig. 1a). All time points starting from 11 a.m. were statistically significantly increased as compared to the first time point at 8 a.m. (8 a.m. vs 11 a.m.: $p<0.05 ; 8$ a.m. vs 1 p.m., 3 p.m., 5 p.m.: $p<0.01 ; n=16)$.

Serum phosphate increased from $2.26 \pm 0.03 \mathrm{mM}, n=16$, in the 9 a.m. samples to $2.64 \pm 0.07 \mathrm{mM}$ at 1 p.m. $(p<0.01$; $n=8)$ and remained constant until 5 p.m. $(2.57 \pm 0.04 \mathrm{mM}$; $n=16)$ (Fig. 1b). Creatinine clearance increased simultaneously from 9 a.m. to 1 p.m. $(p<0.05 ; n=8)$ but decreased again until 5 p.m. (Fig. 1c). The calculated filtered phosphate load thus rose from morning to noon and fell slightly until 5 p.m. (Fig. 1d). These data allowed to also calculate the tubular threshold for phosphate (Tmp). Tmp increased from 9 a.m. to 1 p.m. and leveled off until 5 p.m. (Fig. 1e). Taken together, these data confirm earlier observations in humans and rats showing increased serum and urinary phosphate levels during daytime [17]. The data also indicate that the increased renal phosphate excretion may be related to the higher load and threshold.

\section{Unaltered NaPi-IIa protein expression in the BBM}

To evaluate if reduced expression of $\mathrm{NaPi}$-IIa protein might contribute to the increase in renal phosphate excretion, BBMVs were prepared from kidneys collected in the morning ( 8 a.m.) and afternoon ( 3 p.m.). Figure 2 depicts the relative abundance of NaPi-IIa, i.e., the NaPi-IIa/actin ratio of analyzed band intensities in immunoblots. Our data did not reveal any significant change in NaPi-IIa abundance during the period investigated $(n=8)$. All data were normalized against the mean of $\mathrm{NaPi}$-IIa/actin ratios measured in rats that were killed in the morning.

To assess possible changes in NaPi-IIa distribution within kidney cortex and proximal tubule cells, kidneys were also analyzed immunohistochemically. Screening

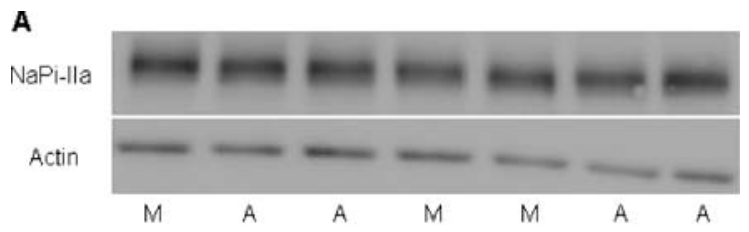

B

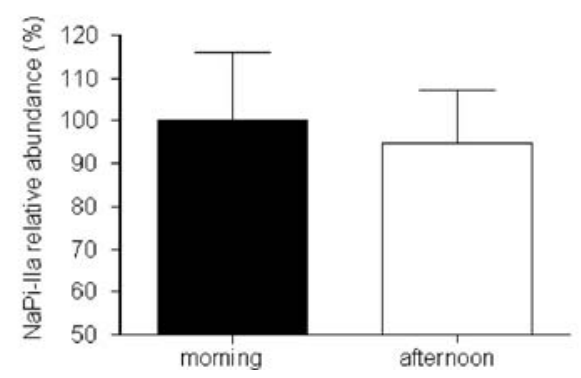

Fig. 2 Unchanged abundance of NaPi-IIa (NaPi-IIa/actin ratio) in BBM. BBMVs were prepared from kidneys collected in the morning $(7.30-8$ a.m.) and afternoon (2.30-3 p.m.) and analyzed by immunoblotting against $\mathrm{NaPi}-\mathrm{II} a$ and actin. a A representative immunoblot of a subset of kidneys is shown; $M$ Morning samples, $A$ afternoon samples. b Intensities of NaPi-IIa and corresponding actin bands were quantified and NaPi-IIa/actin ratios calculated. Data were normalized against the mean of $\mathrm{NaPi}$-IIa/actin ratios determined in kidneys that had been removed in the morning $(=100 \%)$; no significant change was found; $n=8$

several rats of both time groups showed the normal $\mathrm{NaPi}$-IIa expression pattern with increased immunofluorescence in juxtamedullar areas, but which decreased in superficial areas of kidney cortex. Also, no difference in the subcellular localization of NaPi-IIa was evident at higher magnification (Fig. 3). NaPi-IIa-related staining (green) was seen mainly in the BBM (stained with phalloidin-rhodamine in red) and did not change from morning to afternoon.

Decreased sodium-dependent phosphate transport into BBMV

Next, we tested whether the observed diurnal increase in fractional renal phosphate excretion is accompanied by decreased sodium-dependent phosphate transport activity in the proximal tubular BBM. BBMVs were prepared from kidneys removed in the morning and afternoon. As depicted in Fig. $4,{ }^{32} \mathrm{P}$ uptakes were reduced by $14.1 \pm$ $3.3 \%(p<0.01 ; n=4)$ in BBMV from kidneys that had been sampled in the afternoon compared to those in the morning. Rats of both groups that had been killed in 1 day were analyzed within one uptake experiment. Data were normalized to the mean of ${ }^{32} \mathrm{P}$ uptakes measured in kidneys that were collected in the morning.

\section{Discussion}

The data presented in this study demonstrate that renal phosphate excretion increases massively during a 9-h observation period from morning to late afternoon. It is 

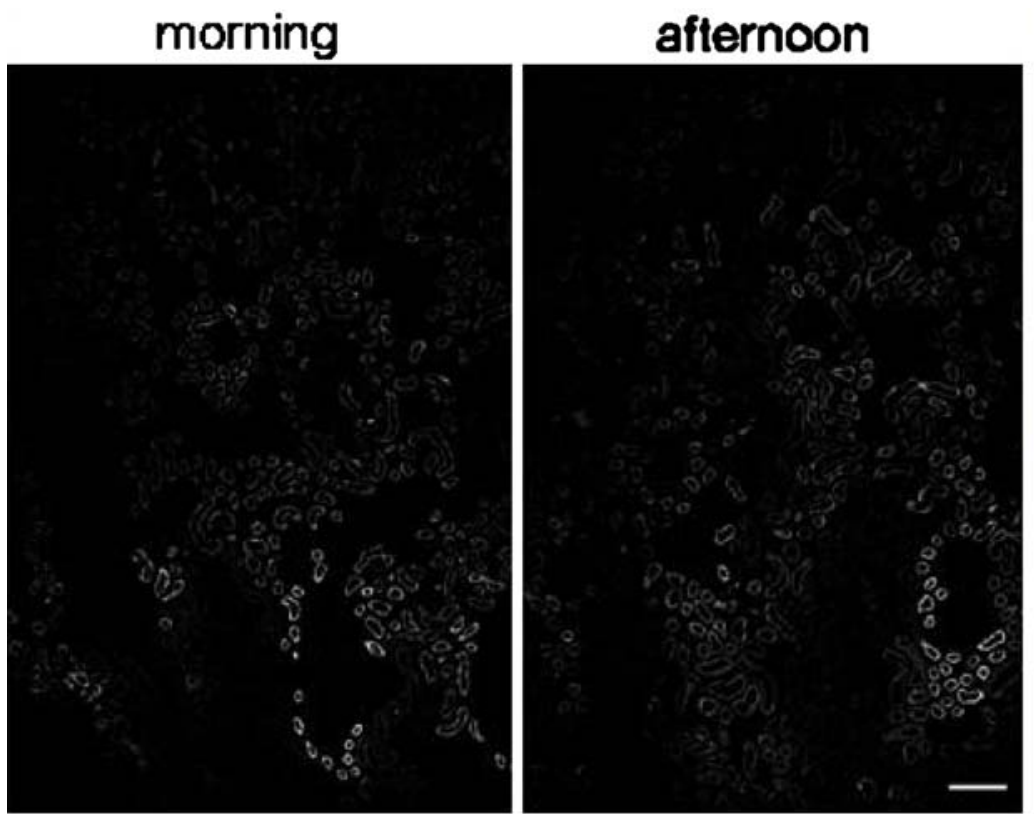

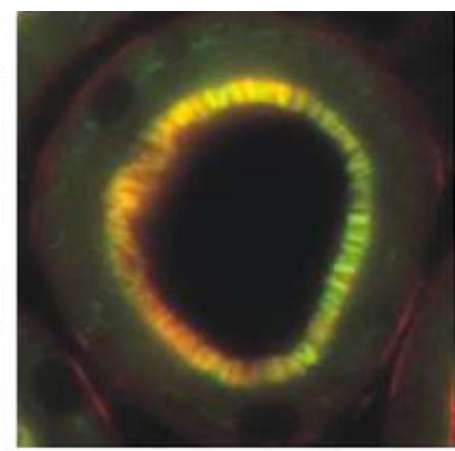

morning

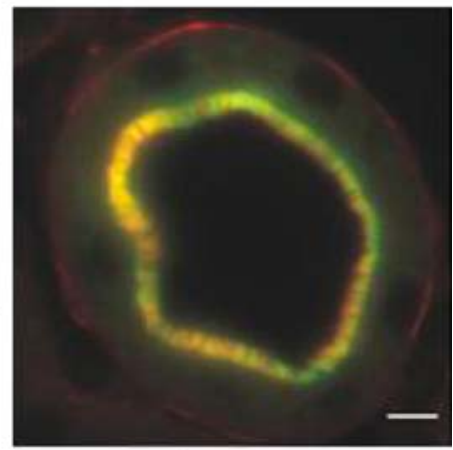

afternoon
Fig. 3 Immunohistochemical analysis of $\mathrm{NaPi}$-IIa protein expression in kidney slices. Rat kidneys were fixed by perfusion at 7:30-8 a.m. and at 2.30-3 p.m. a Representative overviews of renal cortex segments that have been stained for NaPi-IIa. NaPi-IIa-related immunoreactivity is visible in the apical BBMs of proximal tubular cells with a higher intensity of staining in juxtamedullary nephrons. No evidence for differential expression was seen in kidneys fixed either in the morning

interesting to note that phosphaturia is preceded by an increase in serum phosphate, in glomerular filtration rate (inferred from creatinine clearance), filtered phosphate load, and tubular phosphate threshold. All these factors increased between 9 a.m. to 1 p.m. whereas the main rise in renal phosphate excretion occurred only after 11 a.m. Investigating the variations in NaPi-Ila protein abundance or subcellular localization indicates that changes in NaPiIIa represent most likely not a relevant mechanism for

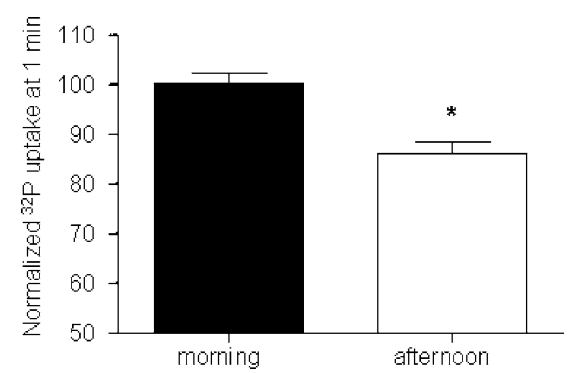

Fig. 4 Sodium-dependent phosphate uptakes into BBMVs. Rats were killed at 7.30-8 a.m. and at 2.30-3 p.m. BBMVs were prepared, and uptake of sodium-dependent phosphate transport $\left({ }^{32} \mathrm{P}\right)$ was measured at $1 \mathrm{~min}$. Data were normalized against the mean of ${ }^{32} \mathrm{P}$ uptakes obtained in kidneys that had been removed in the morning. Uptakes were significantly reduced in kidneys collected in the afternoon vs morning kidneys; $p<0.01 ; n=4$ or afternoon. Original magnification $40 \times$. b Cross section of single proximal tubules from juxtamedullary nephrons was stained for $\mathrm{NaPi}$ IIa (green) and actin (red) as marker for the BBM. Yellow overlap indicates localization of NaPi-IIa in the BBM. The subcellular localization of NaPi-IIa staining was not altered from morning to afternoon. Original magnification $600 \times$

exerting the diurnal increase in renal phosphate excretion. Even though it cannot be excluded that minor changes in NaPi-IIa abundance and localization occurred, but remained undetected, the magnitude of the observed phosphaturia would require a massive downregulation or inhibition of NaPi-IIa if changes in NaPi-IIa were the sole reason. Phosphaturia increased from close to $1 \%$ in the morning to approximately $9 \%$ in the afternoon, large enough that detectable changes in NaPi-IIa abundance could be expected.

PTH is well established to cause phosphaturia, and readily detectable NaPi-IIa downregulation in immunoblots and immunohistochemistry has been reported frequently (reviewed in the study of Murer et al. [21, 22]). Renal phosphate excretion and NaPi-IIa abundance have not been measured in parallel in rats in these studies. However, Zhao and Tenenhouse [31] reported a three- to fourfold relative increase in phosphate excretion after PTH injection in mice together with a $50 \%$ reduction in $\mathrm{Na}^{+}-$ dependent $\mathrm{P}_{\mathrm{i}}$ uptake into BBMV. NaPi-IIa BBM content after PTH treatment was reduced as expected by about 30 $40 \%$. Thus, it is highly unlikely that an increase in the fractional renal phosphate excretion in the range observed in this study could not be detected as a change in NaPi-IIa protein abundance in BBMV if downregulation of NaPi-IIa abundance was the major mechanism. 
Dissociations of renal phosphate excretion and NaPi-IIa protein abundance have been reported previously. Indirect evidence was published by Kempson [13], who reported a fivefold higher apparent Michaelis constant $\left(K_{\mathrm{m}}\right)$ of sodium-dependent phosphate transport in BBMV from fasted rats after a low phosphate diet vs rats continued on a low phosphate diet. The apparent $V_{\max }$ did not change significantly. This finding would argue against a change in NaPi-IIa abundance in this setting as an explanation for altered renal phosphate excretion. However, whether alterations in $K_{\mathrm{m}}$ play a significant role in regulation of phosphate reabsorption remains unclear.

Chronic administration of dihydroxycholecalciferol $\left[1,25(\mathrm{OH})_{2} \mathrm{D}\right]$ has been shown to lower NaPi-IIa BBM protein expression in face of unchanged renal phosphate excretion [7]. Also, chronically potassium-deficient rats, on the other hand, have been reported to exhibit increased urinary phosphate excretion, decreased sodium-dependent BBMV phosphate transport, but increased BBM NaPi-IIa protein abundance [30]. The authors related their findings to altered membrane fluidity or lipid composition [10].

$\mathrm{NaPi}$-IIa abundance does not also fully explain the time course of renal phosphate excretion after PTH administration to parathyroidectomized rats [8]. NaPi-IIa protein abundance remained reduced up to $4 \mathrm{~h}$ after a PTH bolus injection, although renal phosphate excretion had already recovered to control levels $2 \mathrm{~h}$ after PTH injection.

We found slightly but significantly reduced sodiumdependent phosphate transport activity in BBMV derived from kidneys that were removed in the afternoon in spite of unchanged NaPi-IIa protein abundance. Differential regulation of other proximal tubular phosphate transporters, either still unknown or recently identified, such as the sodium-dependent phosphate transporter IIc (NaPi-IIc), may account in part for our observations [18, 25]. An alternative but less plausible explanation would be that, in addition to the well-described regulation via BBM abundance, NaPi-IIa activity may also be regulated functionally.

Taken together, these findings suggest that renal phosphate excretion and NaPi-IIa abundance and activity may dissociate and that other additional factors determine overall renal phosphate excretion. Our data indicate that the filtered load and the tubular threshold for phosphate represent such factors leading to changes in renal phosphate excretion in the face of little or no changes in NaPiIIa abundance and activity.

In summary, the diurnal increase in renal phosphate excretion in rats is not mediated by apparent changes in $\mathrm{NaPi}$-IIa BBM protein abundance and localization. In contrast, changes in tubular phosphate load and tubular threshold for reabsorption appear to suffice to explain the massive phosphaturia observed during the day. It remains to be investigated if regulation of other BBM-located sodium-phosphate cotransporters such as NaPi-IIc may contribute at least in part to the observed phosphaturia.
Acknowledgements Bernhard Bielesz was a participant of the postgraduate course in Experimental Medicine and Biology, Zurich, Switzerland, and wishes to deeply acknowledge the generous support from the Swiss National Science Foundation, grant no. 31000066662. This work was further supported by grants from the Swiss National Science Foundation to H. Murer (31-65397.01) and from the Sixth European Framework EuReGene Project (005085) to C.A. Wagner and $\mathrm{H}$. Murer.

\section{References}

1. Ahmad AM, Hopkins MT, Fraser WD, Ooi CG, Durham BH, Vora JP (2003) Parathyroid hormone secretory pattern, circulating activity, and effect on bone turnover in adult growth hormone deficiency. Bone 32:170-179

2. Biber J, Stieger B, Haase W, Murer H (1981) A high yield preparation for rat kidney brush border membranes. Different behaviour of lysosomal markers. Biochim Biophys Acta 647:169-176

3. Brodehl J, Krause A, Hoyer PF (1988) Assessment of maximal tubular phosphate reabsorption: comparison of direct measurement with the nomogram of Bijvoet. Pediatr Nephrol 2:183-189

4. Carruthers BM, Copp DH, McIntosh HW (1964) Diurnal variation in urinary excretion of calcium and phosphate and its relation to blood levels. J Lab Clin Med 63:959-968

5. Custer M, Lotscher M, Biber J, Murer H, Kaissling B (1994) Expression of Na-P(i) cotransport in rat kidney: localization by RTPCR and immunohistochemistry. Am J Physiol 266:F767-F774

6. Dawson TP, Gandhi R, Le Hir M, Kaissling B (1989) Ecto5 -nucleotidase: localization in rat kidney by light microscopic histochemical and immunohistochemical methods. J Histochem Cytochem 37:39-47

7. Friedlaender MM, Wald H, Dranitzki-Elhalel M, Zajicek HK, Levi M, Popovtzer MM (2001) Vitamin D reduces renal NaPi-2 in PTH-infused rats: complexity of vitamin D action on renal P (i) handling. Am J Physiol Renal Physiol 281:F428-F433

8. Friedlaender MM, Wald H, Dranitzky-Elhalel M, Levi M, Popovtzer MM (2004) Recovery of renal tubule phosphate reabsorption despite reduced levels of sodium-phosphate transporter. Eur J Endocrinol 151:797-801

9. Halloran BP, Portale AA, Castro M, Morris RC Jr, Goldsmith RS (1985) Serum concentration of 1,25-dihydroxyvitamin D in the human: diurnal variation. J Clin Endocrinol Metab 60:1104-1110

10. Inoue M, Digman MA, Cheng M, Breusegem SY, Halaihel N, Sorribas V, Mantulin WW, Gratton E, Barry NP, Levi M (2004) Partitioning of NaPi cotransporter in cholesterol-, sphingomyelin-, and glycosphingolipid-enriched membrane domains modulates NaPi protein diffusion, clustering, and activity. J Biol Chem 279:49160-49171

11. Jubiz W, Canterbury JM, Reiss E, Tyler FH (1972) Circadian rhythm in serum parathyroid hormone concentration in human subjects: correlation with serum calcium, phosphate, albumin, and growth hormone levels. J Clin Invest 51:2040-2046

12. Kemp GJ, Blumsohn A, Morris BW (1992) Circadian changes in plasma phosphate concentration, urinary phosphate excretion, and cellular phosphate shifts. Clin Chem 38:400-402

13. Kempson SA (1985) Effects of fasting compared to low phosphorus diet on the kinetics of phosphate transport by renal brush-border membranes. Biochim Biophys Acta 815:85-90

14. Kishikawa T, Takahashi H, Shimazawa E, Ogata E (1980) Diurnal changes in calcium and phosphate metabolism in rats. Horm Metab Res 12:545-551

15. Laemmli UK (1970) Cleavage of structural proteins during the assembly of the head of bacteriophage T4. Nature 227:680-685

16. Markowitz M, Rotkin L, Rosen JF (1981) Circadian rhythms of blood minerals in humans. Science 213:672-674

17. Mills JN (1966) Human circadian rhythms. Physiol Rev $46: 128-171$ 
18. Miyamoto K, Segawa H, Ito M, Kuwahata M (2004) Physiological regulation of renal sodium-dependent phosphate cotransporters. Jpn J Physiol 54:93-102

19. Murer H, Biber J (1997) A molecular view of proximal tubular inorganic phosphate $(\mathrm{Pi})$ reabsorption and of its regulation. Pflugers Arch 433:379-389

20. Murer H, Evers C, Stoll R, Kinne R (1977) The effect of parathyroid hormone (PTH) and dietary phosphate on the sodiumdependent phosphate transport system located in the rat renal brush border membrane. Curr Probl Clin Biochem 8:455-462

21. Murer H, Forster I, Hernando N, Lambert G, Traebert M, Biber J (1999) Posttranscriptional regulation of the proximal tubule NaPi-II transporter in response to PTH and dietary P(i). Am J Physiol 277:F676-F684

22. Murer H, Hernando N, Forster I, Biber J (2000) Proximal tubular phosphate reabsorption: molecular mechanisms. Physiol Rev 80:1373-1409

23. Murer H, Hernando N, Forster I, Biber J (2003) Regulation of $\mathrm{Na} / \mathrm{Pi}$ transporter in the proximal tubule. Annu Rev Physiol 65:531-542

24. Roelfsema F, van der Heide D, Smeenk D (1980) Circadian rhythms of urinary electrolyte excretion in freely moving rats. Life Sci 27:2303-2309

25. Segawa H, Kaneko I, Takahashi A, Kuwahata M, Ito M, Ohkido I, Tatsumi S, Miyamoto K (2002) Growth-related renal type II Na/Pi cotransporter. J Biol Chem 277:19665-19672
26. Shinoda H, Seto H (1985) Diurnal rhythms in calcium and phosphate metabolism in rodents and their relations to lighting and feeding schedules. Miner Electrolyte Metab 11:158-166

27. Talmage RV, Roycroft JH, Anderson JJ (1975) Daily fluctuations in plasma calcium, phosphate, and their radionuclide concentrations in the rat. Calcif Tissue Res 17:91-102

28. Towbin H, Staehelin T, Gordon J (1979) Electrophoretic transfer of proteins from polyacrylamide gels to nitrocellulose sheets: procedure and some applications. Proc Natl Acad Sci U S A 76:4350-4354

29. Vesely DL, Sothern RB, Scheving LE, Bremner FW, Third JL, McCormick JB, Dawson S, Kahn S, Augustine G, Ryan M, Greco J, Nemchausky BA, Shirazi P, Kanabrocki EL (1996) Circadian relationships between circulating atrial natriuretic peptides and serum calcium and phosphate in healthy humans. Metabolism 45:1021-1028

30. Zajicek HK, Wang H, Puttaparthi K, Halaihel N, Markovich D, Shayman J, Beliveau R, Wilson P, Rogers T, Levi M (2001) Glycosphingolipids modulate renal phosphate transport in potassium deficiency. Kidney Int 60:694-704

31. Zhao N, Tenenhouse HS (2000) Npt2 gene disruption confers resistance to the inhibitory action of parathyroid hormone on renal sodium-phosphate cotransport. Endocrinology 141:2159-2165 